\section{(6) OPEN ACCESS}

\begin{abstract}
- Additional material is published online only. To view please visit the journal online (http://dx.doi.org/10.1136/ thoraxjnl-2018-212102).
\end{abstract}

For numbered affiliations see end of article.

\section{Correspondence to} Professor A John Simpson, Institute of Cellular Medicine, Newcastle University, Newcastle upon Tyne NE2 4HH, UK; j.simpson@ncl.ac.uk

PD and WF contributed equally. IAF and AJS contributed equally.

Received 22 May 2018 Revised 25 October 2018 Accepted 12 November 2018 Published Online First 4 January 2019

\section{Check for updates}

(C) Author(s) (or their employer(s)) 2019. Re-use permitted under CC BY-NC. No commercial re-use. See rights and permissions. Published by BMJ.

To cite: Dutta P, Funston W, Mossop $\mathrm{H}$, et al. Thorax 2019:74:346-353.

\title{
Randomised, double-blind, placebo-controlled pilot trial of omeprazole in idiopathic pulmonary fibrosis
}

\author{
Prosenjit Dutta, ${ }^{1}$ Wendy Funston, ${ }^{1}$ Helen Mossop, ${ }^{2}$ Vicky Ryan, ${ }^{2}$ Rhys Jones, \\ Rebecca Forbes, ${ }^{3}$ Shilpi Sen, ${ }^{4}$ Jeffrey Pearson, ${ }^{5}$ S Michael Griffin, ${ }^{6}$ Jaclyn A Smith, ${ }^{4,7}$ \\ Christopher Ward, ${ }^{1}$ lan A Forrest, ${ }^{8}$ A John Simpson ${ }^{1,8}$
}

\begin{abstract}
Background Cough is a common, disabling symptom of idiopathic pulmonary fibrosis (IPF), which may be exacerbated by acid reflux. Inhibiting gastric acid secretion could potentially reduce cough. This study aimed to determine the feasibility of a larger, multicentre trial of omeprazole for cough in IPF, to assess safety and to quantify cough.

Methods Single-centre, double-blind, randomised, placebo-controlled pilot trial of the proton pump inhibitor (PPI) omeprazole (20 mg twice daily for 3 months) in patients with IPF. Primary objectives were to assess feasibility and acceptability of trial procedures. The primary clinical outcome was cough frequency.

Results Forty-five participants were randomised (23 to omeprazole, 22 to placebo), with 40 (20 in each group) having cough monitoring before and after treatment. 280 patients were screened to yield these numbers, with barriers to discontinuing antacids the single biggest reason for non-recruitment. Recruitment averaged 1.5 participants per month. Geometric mean cough frequency at the end of treatment, adjusted for baseline, was 39.1\% lower (95\% Cl 66.0\% lower to $9.3 \%$ higher) in the omeprazole group compared with placebo. Omeprazole was well tolerated and adverse event profiles were similar in both groups, although there was a small excess of lower respiratory tract infection and a small fall in forced expiratory volume and forced

\section{Key messages}

What is the key question?

- Are there sufficient grounds to justify larger randomised controlled trials of proton pump inhibitors in idiopathic pulmonary fibrosis (IPF) on the basis of feasibility and safety, and is cough a potential outcome measure for efficacy?

What is the bottom line?

- Omeprazole is well-tolerated and may have a positive effect on cough in IPF, suggesting larger trials are feasible but they will require screening of large numbers of patients and careful monitoring of adverse events, with particular reference to frequency of respiratory infections and changes in lung function.

Why read on?

- Controversy has surrounded the dual issues of whether proton pump inhibitors are beneficial in IPF and whether symptom-centred primary outcome measures can be usefully integrated when assessing new treatments for IPF-this study provides positive new information addressing both questions and outlines challenges in planning larger, definitive trials.
\end{abstract} vital capacity associated with omeprazole.

Conclusions A large randomised controlled trial of PPIs for cough in IPF appears feasible and justified but should address barriers to randomisation and incorporate safety assessments in relation to respiratory infection and changes in lung function.

\section{INTRODUCTION}

Idiopathic pulmonary fibrosis (IPF) is a chronic, fibrosing interstitial lung disease (ILD) characterised by progressive dyspnoea and cough. Chronic cough is a major and disabling symptom affecting up to $80 \%$ of patients with IPF, ${ }^{1}$ and a strong correlation has been demonstrated between objective cough frequency and health-related quality of life. $^{2}$ Nevertheless, treatment of cough associated with IPF remains notoriously difficult and conventional antitussive treatments have limited therapeutic effect. ${ }^{3}$

Gastro-oesophageal reflux disease (GORD) is more prevalent in patients with IPF compared with the general population and those with other chronic lung diseases, ${ }^{4}$ and a large proportion of patients with IPF have asymptomatic reflux disease. ${ }^{5-7}$ GORD has been postulated as a contributory factor to chronic cough in IPF and to the overall pathogenesis and progression of the disease. The use of antacid strategies such as proton pump inhibitors (PPIs) has therefore been commonplace in many countries and conditional recommendations for antacid treatment have been incorporated into current guidelines. ${ }^{8}$

Despite the frequent use of antacid therapy in this population, there are currently no large, randomised controlled trials (RCTs) evaluating the efficacy of PPIs in relation to cough frequency in IPF, and assessing their safety. This pilot trial was therefore designed to assess the feasibility of an RCT of omeprazole in IPF, with particular reference to safety and whether any potential effect on cough would emerge. Ultimately, the aim of the study was to determine whether a larger definitive multicentre trial is practicable and worthwhile and, 
if so, to define possible barriers that should be addressed during trial design.

\section{METHODS}

\section{Study design and participants}

A single-centre, double-blind, placebo-controlled, randomised pilot trial of omeprazole in patients with IPF was conducted at the Newcastle upon Tyne Hospitals NHS Foundation Trust. Participants aged 40-85 years with IPF were recruited from the ILD clinic at the Royal Victoria Infirmary, Newcastle upon Tyne, UK. Patients could also be identified and referred for participation from one of six Participant Identification Centres (PICs) by their treating clinicians.

A pragmatic clinical definition of IPF was used, in which participants were required to fulfil all of the following criteria: IPF considered the most likely diagnosis by the regional ILD multidisciplinary team; history of cough; radiological features of honeycombing in a predominantly basal and subpleural distribution on high-resolution CT (HRCT) scanning; bibasal inspiratory crackles on auscultation and features of a restrictive ventilatory defect (vital capacity (VC) $<90 \%$ predicted and/or transfer factor for carbon monoxide (TLCo) $<90 \%$ predicted). ${ }^{9}{ }^{10}$ Due to possible interactions with omeprazole, participants were excluded if they were taking warfarin, diazepam, phenytoin or ketoconazole. Participants taking active treatments for IPF (eg, pirfenidone or nintedanib) had to have been taking these for at least 4 weeks before entry to the study. Other exclusion criteria included upper or lower respiratory tract infection or exacerbation of IPF in the 4 weeks prior to starting trial treatment, or a history of hepatic cirrhosis.

Patients taking antacids, prokinetics or raft alginates were eligible if they had been off these treatments for at least 2 weeks. Patients taking a regular PPI who wished to take part were eligible if they provided written informed consent to a 2 -week trial period off treatment, following agreement with their general practitioner, and if the trial was tolerated and completed with no significant return of upper gastrointestinal (GI) symptoms such as reflux, indigestion or heartburn. Patients were asked to refrain from using over the counter antacids and to contact the study team if these were used, with a view to withdrawal from the trial. The online supplementary section contains full details of the study schedule (in Table S1), and of the trial's inclusion and exclusion criteria. All participants provided written informed consent.

\section{Study drug, randomisation and blinding}

The study compared omeprazole, a substituted benzimidazole PPI, with placebo. Participants were randomised 1:1 to receive omeprazole $20 \mathrm{mg}$ or matching placebo, to be taken orally twice daily before food for 90 days; however, planned treatment duration between 76 and 90 days was permitted. All other aspects of usual patient care were delivered to participants prior to, during and after completion of the study. Randomisation was through a secure password-protected web-based system administered centrally by the Newcastle Clinical Trials Unit. Computer-generated random permuted blocks of size 12 were used. The participant, research team, investigators and sponsor were all blinded to allocation. Omeprazole and matched placebo were supplied by Victoria Pharmaceuticals, Royal Hospitals, Belfast, UK and dispensed by the pharmacy at the Royal Victoria Infirmary, Newcastle upon Tyne, UK.

\section{Procedures}

Participant demographics, medical comorbidities, smoking history and concurrent medications were recorded at screening.
After informed consent was obtained, baseline assessments included physical examination, pulmonary function tests (PFTs) and a 6 min walk distance (6MWD). Validated questionnaires to assess cough and reflux symptoms were completed by the participant and included the De Meester reflux-related symptoms questionnaire (DeMRQ) ${ }^{11}$ the Reflux Symptoms Index (RSI) ${ }^{12}$ the Gastrointestinal Quality of Life Index (GIQLI) ${ }^{13}$ and the Leicester Cough Questionnaire (LCQ). ${ }^{14}$ Details of the scoring system for each of the questionnaires are described in the supplemental section. A higher score indicates better health status for the LCQ and GIQLI and the converse is true for the DeMRQ and RSI. Participants were also fitted with an ambulatory cough recorder (Vitalojak, Vitalograph, Buckingham, UK) for 24 hours. $^{2}{ }^{15}$ PFTs, 6MWD, questionnaires and cough sound recording were repeated at the end of the treatment period (ideally these tests were performed on day 88 of the 90-day study medication trial but could be performed at any point from day 76 onwards).

Oesophageal physiology testing was not mandatory for inclusion; however, for those giving consent to oesophageal procedures these comprised high-resolution oesophageal manometry (to measure oesophageal muscular contraction and relaxation of the gastro-oesophageal sphincter) and 24 hours $\mathrm{pH}$-impedance monitoring (to record regurgitation of gastric contents into the oesophagus and participant-reported symptom episodes). Assessments were performed at baseline and at the end of the treatment period. Bronchoscopy with bronchoalveolar lavage (BAL) was also not mandatory for inclusion, but where consent for the procedure was agreed, this was performed at the end of the treatment period. Oesophageal physiology testing and bronchoscopy with standardised BAL were ideally performed on days 89 and 90 of the 90 -day study medication period, respectively but could be performed at any point from day 76 onwards. Details of these procedures are given in the online supplemental section.

Participants reported adverse events using a patient diary card. Episodes of hospitalisation were reported via telephone. All participants were asked to stop the study medication on the day they completed their last study procedure. Remaining study medication was returned to the pharmacy at the end of the trial treatment period and unused supplies were counted.

\section{Outcomes}

Feasibility outcomes comprised the number of eligible patients, the proportion of those willing to take part, the recruitment rate, adherence to trial medication and follow-up and the acceptability of invasive trial procedures.

Clinical outcome measures were collected to interrogate proof of concept with respect to reduction in cough frequency. Twenty-four hours objective cough frequency (total number of coughs/ total recording time in hours) was assessed using an ambulatory cough recorder at baseline and the end of the treatment period. Sound recordings were compressed using validated customwritten software to remove silences, background noise and the majority of speech. ${ }^{16}$ The resulting files were then listened to and cough sounds manually counted. Awake and asleep cough frequencies were calculated.

Additional clinical outcomes included 6MWD and PFTs $\left(\mathrm{FEV}_{1}\right.$, FVC, the $\mathrm{FEV}_{1} / \mathrm{FVC}$ ratio, TLco and Kco (TLco corrected for accessible alveolar volume)). Participant-reported outcomes were the total DeMRQ, RSI, GIQLI and LCQ scores. Domain scores of the LCQ were also explored.

\section{Statistical analysis}

Due to the nature of this pilot study no formal sample size calculation was performed. Recommendations for good practice at 
the time the study was designed suggested that 20-30 participants per treatment group should provide sufficient data to assess the feasibility of a trial, investigate the distribution of outcome measures and estimate with adequate precision SD of key study parameters. ${ }^{17} 18$

Analyses were performed according to a predefined statistical analysis plan (a copy is shown at the end of the online supplemental section). Feasibility outcomes were summarised descriptively. Analysis of all clinical outcomes was performed on a complete-case basis following the intention-to-treat principle. Only observed data were included and there was no imputation of missing data. For daytime, night-time and 24 hours cough frequency, data were analysed following a $\log _{10}$ transformation. A value of 0.1 was added to all night-time cough frequencies prior to transformation due to zero coughs being reported for some participants. On the logarithmic scale, the difference between randomised treatment groups in mean cough frequency at end of treatment was estimated using an analysis of covariance (ANCOVA) model, adjusted for baseline cough frequency. Model estimates and 95\% CIs were then back-transformed to give an estimate of the ratio of objective cough frequency in the omeprazole group compared with the placebo group at the end of treatment. Untransformed secondary clinical outcome measures (excluding DeMRQ) were analysed using similar ANCOVA models, adjusted for the appropriate baseline value.

Safety data were summarised, for each event, by the worst grade reported per participant over the trial treatment period. Participants were included in safety analyses if they had taken at least one dose of study drug (omeprazole or placebo). Oesophageal physiology, bronchoscopy and BAL measures were summarised descriptively.

The trial database was locked for final analysis on 16 June 2017. All analyses were performed using Stata V.14.

\section{Regulatory approvals}

The clinical trial authorisation was given by the Medicines \& Healthcare products Regulatory Agency, sponsored by the Newcastle upon Tyne Hospitals NHS Foundation Trust and supported by the (fully UKCRC-registered) Newcastle Clinical Trials Unit. The trial was registered as an International Standard Randomised Controlled Trial (ISRCTN07139948), with the EU Clinical Trials Register (EudraCT 2013-003301-26) and with ClinicalTrials.gov (NCT02085018) prior to the start of the trial. The trial was adopted onto the National Institute for Health Research portfolio (study ID 15732) and overseen by an independent trial steering committee and data monitoring and safety committee.

\section{RESULTS \\ Primary feasibility outcomes}

Between 1 March 2014 and 19 August 2016, 45 patients were recruited, with 23 assigned to receive omeprazole, as illustrated in figure 1 . In total, 280 patients were assessed for eligibility, 159 (57\%) were ineligible or clinically unsuitable and 67 (24\%) declined participation or could not be contacted. The most common reasons for ineligibility were unsuitability for a trial off PPI $(n=34)$, no cough $(n=24)$ and concomitant use of warfarin $(n=20)$. Overall, 25 patients on regular PPI at screening consented to a trial off treatment. Of these, nine experienced a return of upper GI symptoms and were excluded from the study while 16 reported no return of upper GI symptoms and were subsequently randomised (eight to each treatment group). The average recruitment rate was 1.5 participants per month. A total of 13 patients were referred from 6 regional hospitals acting as PIC sites; however, none of these were randomised. Following randomisation, attrition rate was low with 40 participants (89\%)

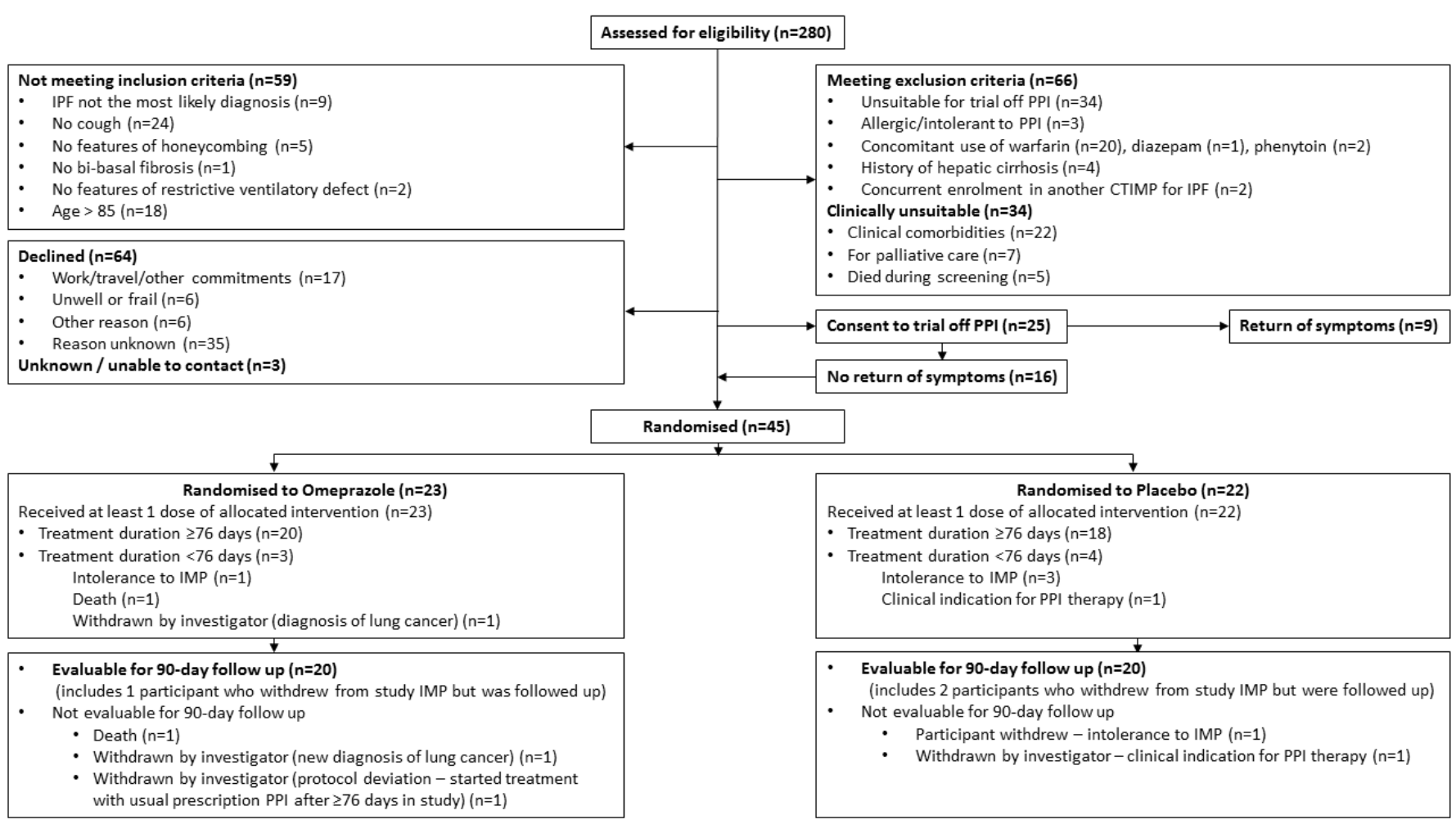

Figure 1 Consolidated Standards of Reporting Trials diagram. IPF, idiopathic pulmonary fibrosis; PPI, proton pump inhibitor; CTIMP, clinical trial of an investigational medicinal product; IMP, investigational medicinal product. 
completing end of treatment cough monitoring visits (although not all participants completed all the other possible study assessments).

Median treatment duration was 86 days (IQR 82-90) in the omeprazole group and 87.5 days (IQR 79-90) in the placebo group. Median tablet return was 14 tablets (IQR 6-25) in the omeprazole group and 21.5 tablets (IQR 2-32) in the placebo group. Overall, seven participants (three omeprazole, four placebo) discontinued treatment early (ie, treatment duration $<76$ days). One patient was withdrawn (from the placebo group) because of a clinical requirement for PPIs, and use of over the counter antacids. No unblinding was required during the study.

All randomised participants consented to the non-invasive trial procedures (including ambulatory cough monitor, questionnaires, walk test and PFTs). Completion rate was high with most participants completing all non-invasive assessments (three participants could not complete PFTs due to poor technique, one amputee could not complete a walk test, one participant was admitted with sepsis and did not complete tests).

In contrast, a relatively small proportion of participants consented to undergo invasive tests (oesophageal manometry, 24 hours oesophageal $\mathrm{pH}$ monitoring and bronchoscopy). Of the 45 participants randomised, 13 consented to 24 hours $\mathrm{pH}$ monitoring, however only 9 completed this at baseline and 6 at end of treatment. Thirteen participants consented to undergo bronchoscopy but only eight completed the assessment.

Baseline demographics and clinical characteristics were approximately balanced between the randomised groups (table 1).

\section{Primary clinical outcome}

Forty participants $(89 \%)$ completed cough monitoring before and after treatment. Baseline geometric mean 24 hours cough frequency (coughs/hour) was 8.2 (95\% CI 5.4 to 12.7 ) and 9.1 (95\% CI 6.8 to 12.2$)$ in the omeprazole and placebo groups, respectively. There was a greater reduction in 24 hours cough frequency in the omeprazole group compared with placebo. At the end of the study geometric mean was 4.6 per hour $(95 \% \mathrm{CI}$ 2.4 to 8.7$)$ in the omeprazole group and 8.3 per hour $(95 \%$ CI 5.3 to 12.9 ) in the placebo group. Geometric mean cough frequency at the end of treatment, adjusted for baseline, was $39.1 \%$ lower (95\% CI $66.0 \%$ lower to $9.3 \%$ higher) in the omeprazole group compared with placebo (table 2). Similar results were observed for the relative difference in awake/daytime and asleep/nighttime cough frequency (table 2). Individual participant changes in 24 hours, awake/daytime and asleep/night-time objective cough frequency are illustrated in figure 2. Absolute cough frequency for individuals, before and after treatment, is shown in online supplementary figure S1.

\section{Secondary outcomes}

Participant-reported symptoms of cough (using the LCQ) and reflux (using the DeMRQ, the GIQLI and the RSI) were assessed. Overall, there was no clinically meaningful difference for participant-reported outcome measures between baseline and end of treatment in either group. The between-group differences at end of treatment for each of the questionnaires are summarised in table 3.

Functional status (assessed by PFTs and 6MWD) was obtained at baseline and end of treatment for each group (table 4). No clinically meaningful difference in PFTs or 6MWD values was observed between baseline and end of treatment for either group.
Table 1 Baseline demographics and clinical characteristics

\begin{tabular}{|c|c|c|}
\hline & Omeprazole $(n=23)$ & Placebo $(n=22)$ \\
\hline Female & $4(17 \%)$ & $6(27 \%)$ \\
\hline Male & $19(83 \%)$ & $16(73 \%)$ \\
\hline Age (years) & $71.3(6.7)$ & $71(7.3)$ \\
\hline \multicolumn{3}{|l|}{ Smoking history } \\
\hline Never smoked & $5(22 \%)$ & $5(23 \%)$ \\
\hline Ex-smoker & $18(78 \%)$ & $16(73 \%)$ \\
\hline Current smoker & $0(0 \%)$ & $1(5 \%)$ \\
\hline Pack years* & $13.0(7.5-20.0)$ & $15.0(7.0-36.0)$ \\
\hline BMI $\left(\mathrm{kg} / \mathrm{m}^{2}\right)$ & $28.9(3.8)$ & $29.6(6.0)$ \\
\hline Concomitant pirfenidone & 8 & 10 \\
\hline Concomitant nintedanib & 0 & 2 \\
\hline Concomitant prednisolone & 0 & 4 \\
\hline Respiratory rate (breaths/min) & $21.5(3.5)$ & $22.4(3.8)$ \\
\hline $\mathrm{FEV}_{1}(\%$ predicted $)$ & $76.9(15.4)$ & $78.4(18.4)$ \\
\hline FVC (\% predicted) & $73.1(17.1)$ & 77.9 (17.6) \\
\hline Kco (\% predicted) & $87.3(20.9)$ & $83.0(22.0)$ \\
\hline TLco (\% predicted) & $49.5(15.7)$ & $48.4(16.0)$ \\
\hline $6 \mathrm{MWD}(\mathrm{m})$ & $416.5(296.5-485.0)$ & $372.5(307.6-450.0)$ \\
\hline DeMRQ & $0.9(0.8)$ & $1.5(1.4)$ \\
\hline GIQLI & $106.3(17.9)$ & $104.8(17.8)$ \\
\hline LCQ & $15.1(3.2)$ & $15.4(3.2)$ \\
\hline RSI & $14.3(9.6)$ & $17.1(9.0)$ \\
\hline Coughs/hour-24hours & $9.6(4.2-18.3)$ & $8.9(6.8-12.8)$ \\
\hline Coughs/hour—daytime & $13.4(6.0-24.3)$ & $11.6(8.5-17.4)$ \\
\hline Coughs/hour-night-time & $2.1(0.5-6.9)$ & $2.6(0.8-9.0)$ \\
\hline
\end{tabular}

Data are presented as $n(\%)$, mean (standard deviation (SD)) or median (IQR).

* Pack years are shown for participants who were current or ex-smokers and for whom the number of pack years was known ( $n=16$ in the omeprazole group and $n=15$ in the placebo group).

tThe doses received by the 4 patients were $4 \mathrm{mg}, 6 \mathrm{mg}, 10 \mathrm{mg}$ or $15 \mathrm{mg}$ per day. 6MWD was

performed in 21 of the 22 participants in the placebo group and in all 23 participants in the omeprazole group.

6MWD, 6 min walk distance; BMI, body mass index; DeMRQ, De Meester reflux-related symptoms questionnaire; GIQLI, Gastrointestinal Quality of Life Index; LCQ, Leicester Cough Questionnaire; RSI, Reflux Symptoms Index; TLco, transfer factor for carbon monoxide

Due to the small numbers of participants consenting to and completing the invasive tests (oesophageal manometry, 24-hours $\mathrm{pH}$ monitoring and bronchoscopy), few data were obtained (see online supplementary table S2-S5).

\section{Safety}

In total, nine serious adverse events were reported across eight participants, four allocated to omeprazole and four to placebo. Relatedness to trial treatment was judged by the participant's clinician, who was blind to treatment allocation. Three participants (one omeprazole, two placebo) reported moderate-to-severe abdominal pain, which was deemed an expected serious adverse reaction. Two participants died and a further three were admitted to hospital while taking part in the study-all were considered unrelated to trial treatment. Three participants withdrew from trial treatment due to abdominal pain (one omeprazole, two placebo) and one participant allocated to placebo due to hot flushes and fatigue after one dose. Adverse events were reported in 16 participants in the omeprazole group (70\%) and 14 in the placebo group (64\%). Table 5 details adverse events that were reported in more than one participant in the omeprazole group. More complete safety data are found in online supplementary table S6-S8 and show no striking differences between the groups. 
Table 2 Geometric mean cough frequency (coughs/hour) and the ratio (omeprazole:placebo) of geometric means at the end of treatment, adjusted for baseline cough frequency (complete-case analysis set; $n=40$ )

\begin{tabular}{|c|c|c|c|c|c|}
\hline & \multicolumn{2}{|l|}{ Omeprazole $(n=20)$} & \multicolumn{2}{|l|}{ Placebo $(n=20)$} & \multirow{2}{*}{$\begin{array}{l}\text { Ratio of geometric means } \\
\text { at end of treatment, } \\
\text { adjusted for baseline } \\
(95 \% \mathrm{Cl})\end{array}$} \\
\hline & $\begin{array}{l}\text { Baseline } \\
\text { geometric mean }(95 \% \mathrm{CI})\end{array}$ & $\begin{array}{l}\text { End of treatment } \\
\text { geometric mean }(95 \% \mathrm{Cl})\end{array}$ & $\begin{array}{l}\text { Baseline } \\
\text { geometric mean }(95 \% \mathrm{Cl})\end{array}$ & $\begin{array}{l}\text { End of treatment } \\
\text { geometric mean }(95 \% \mathrm{Cl})\end{array}$ & \\
\hline 24 hours & $8.2(5.4$ to 12.7$)$ & 4.6 (2.4 to 8.7$)$ & $9.1(6.8$ to 12.2$)$ & $8.3(5.3$ to 12.9$)$ & 0.609 (0.340 to 1.093$)$ \\
\hline Awake/daytime & $10.8(6.8$ to 17.0$)$ & 6.1 (3.2 to 11.8$)$ & 11.8 (8.9 to 15.8$)$ & $10.7(6.7$ to 17.2$)$ & $0.629(0.354$ to 1.119$)$ \\
\hline Asleep/night-time & 1.7 (0.9 to 3.3$)$ & 1.1 (0.6 to 2.0$)$ & 2.3 (1.1 to 4.7$)$ & 2.1 (1.2 to 3.7$)$ & $0.553(0.267$ to 1.146$)$ \\
\hline
\end{tabular}

\section{DISCUSSION}

While significant advances have been made in slowing disease progression in IPF through the emergence of antifibrotic medications such as pirfenidone and nintedanib, ${ }^{19}{ }^{20}$ there is a pressing need to find solutions for chronic symptom management. Chronic cough is one of the most common, distressing and notoriously difficult symptoms to control in patients with IPF, with a significant impact on quality of life. ${ }^{2}$ Studies evaluating potential therapies for chronic cough in IPF are scarce and have shown limited benefit. ${ }^{121-23}$

The hypothesis that GORD perpetuates disease progression in IPF has led to studies primarily evaluating the impact of PPIs on measures of disease progression and survival. ${ }^{24-27}$ No definitive conclusion has emerged from these valuable studies, in which a range of different methodologies have been employed. To our knowledge, this study represents the first pilot RCT of omeprazole for the treatment of cough in IPF, while a recent pilot RCT

$24 \mathrm{~h}$

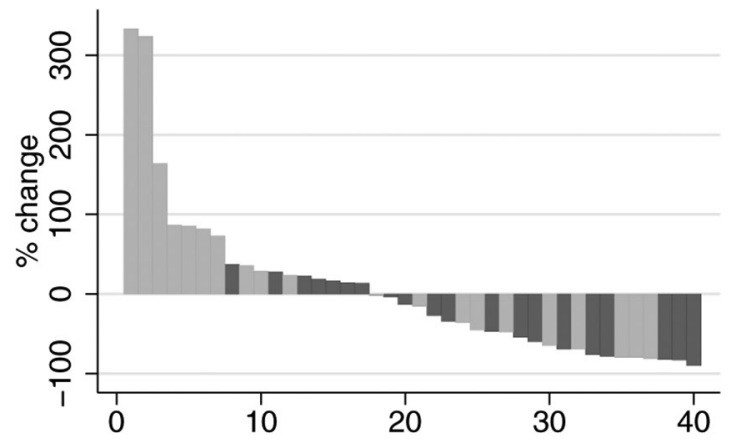

Night-time

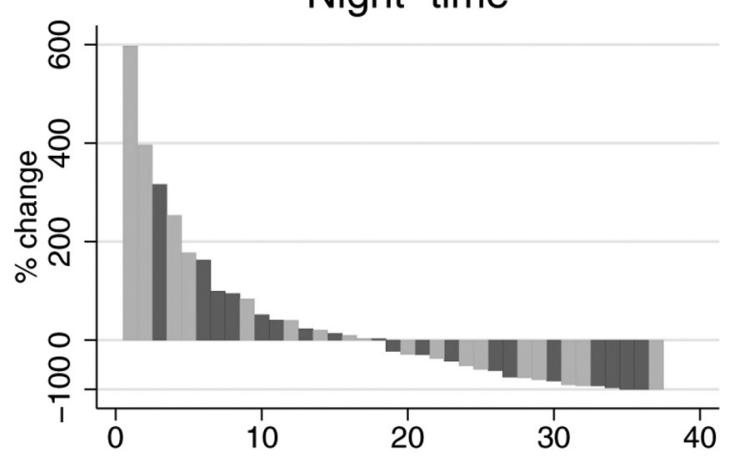

has highlighted the feasibility of performing future trials assessing the value of laparoscopic antireflux surgery in $\mathrm{IPF}^{28}$ Our data suggested that, on average, cough was reduced in the omeprazole group, although this was not statistically significant (as a pilot trial, the study was not designed to have sufficient power to detect statistically significant differences). The magnitude of the observed change in favour of omeprazole is considered clinically important, ${ }^{29}$ and is comparable to the effect size observed in studies reporting clinically meaningful reductions in objective cough frequency with other pharmacological treatments for chronic cough. ${ }^{3031}$

The baseline cough frequency was lower than that reported in previous studies of IPF. The reasons for this are not clear but may relate to the fact that we allowed enrolment of participants with self-reported cough, regardless of severity. On balance, we believe the average reduction in cough frequency associated with omeprazole justifies further assessment of this effect in a larger trial.

Figure 2 Individual participant percentage change in 24 hours, awake (daytime) and asleep (night-time) objective cough frequency (coughs/ hour). Data are shown for the complete-case analysis set, $n=40$. Percentage change $=(($ end of treatment-baseline/baseline $) \times 100)$. Each column on the $x$-axis represents a participant. The night-time graph has $n=37$, as three participants ( 2 placebo, 1 omeprazole) had zero night-time coughs at baseline (end of treatment coughs for these participants were 1.86, 0.32 (placebo) and 0.59 (omeprazole)). 
Table 3 Between-group differences in participant-reported outcome measures

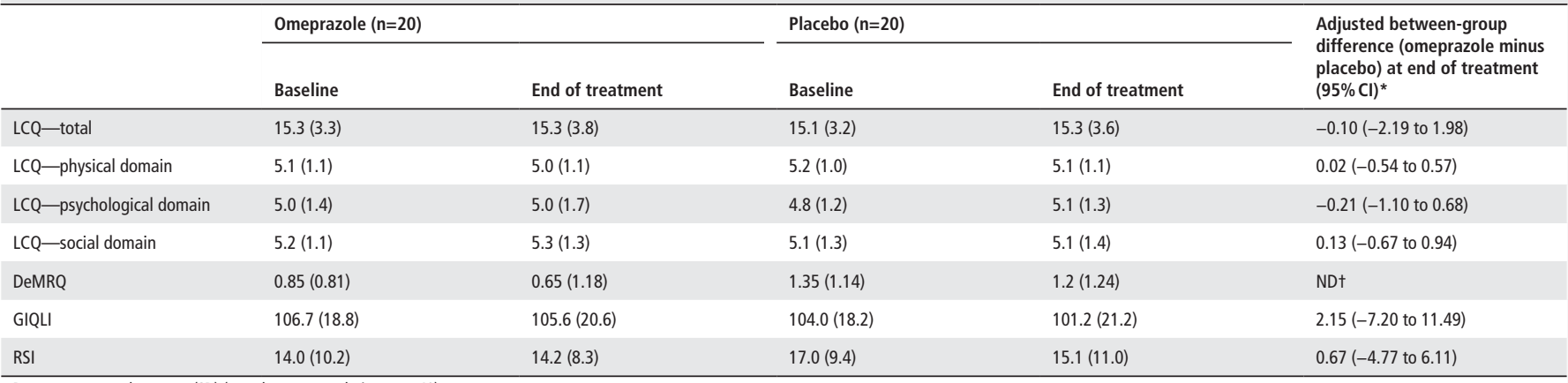

Data are presented as mean (SD) (complete-case analysis set; $n=40$ ).

*Between-group difference at end of treatment was calculated using ANCOVA, adjusted for baseline value and shows the change relative to that in the placebo group. ND: not done

† Not done as model assumptions not satisfied. For LCQ and GIQLI a higher score indicates improvement, for DeMRQ and RSI a lower score indicates improvement

DeMRQ, De Meester reflux-related symptoms questionnaire; GIQLI, Gastrointestinal Quality of Life Index; LCQ, Leicester Cough Questionnaire; RSI, Reflux Symptoms Index.

Omeprazole appears to have been well tolerated in this study. Stomach acid is thought to eradicate ingested bacteria, leading to the concern that PPIs may promote retention of microbes in gastric fluid, which in turn can be micro-aspirated into the lungs. Higher rates of lower respiratory tract infection have been described in patients prescribed PPIs, although some of the data pertain to post hoc analyses, with the inherent limitations associated with this approach. ${ }^{26} 2{ }^{32-34}$ While adverse events were generally evenly distributed in the two groups, lower respiratory tract infections were twice as common in the omeprazole group, although the numbers involved (six in the omeprazole group, three in the placebo group) raise the possibility that the observation arose by chance. Accurate discrimination of true alveolar infection from the natural history of progressive fibrosis is difficult in IPF and infection was assessed on clinical suspicion in this study, without microbiological confirmation. Interestingly, $\mathrm{FEV}_{1}$ and FVC were significantly lower in the omeprazole group (table 4). However, an apparent trend in the opposite direction (ie, favouring omeprazole) was observed for Kco (table 4). On balance, PPIs appear to be well tolerated in IPF, but future trials should monitor lung function and rates of lower respiratory tract infection.

If it is accepted that tolerance of omeprazole, and its potential to reduce cough, justify pursuit of a larger RCT, the next question is whether such a study is likely to be feasible? On the basis of our evidence we believe such a study is eminently feasible, although several issues will require consideration. These will include taking account of the proportion of patients already taking long-term PPIs. There are few indications for long-term PPIs, so aligning patients' prescriptions with guidelines should be expected to allow their discontinuation. The welcome proliferation of available clinical trials in IPF may also reduce the potential pool of patients available to a larger trial of PPIs in IPF. Our clinic is a regional referral centre for antifibrotic drugs and therefore sees a high volume of patients, and even under these circumstances we took two and a half years to generate

Table 4 Pulmonary function tests and 6MWD at baseline and end of treatment

\begin{tabular}{|c|c|c|c|c|c|}
\hline & \multicolumn{2}{|c|}{ Omeprazole $(n=20)$} & \multicolumn{2}{|c|}{ Placebo $(n=20)$} & \multirow{2}{*}{$\begin{array}{l}\text { Adjusted between group } \\
\text { difference (omeprazole minus } \\
\text { placebo) at end of treatment } \\
(95 \% \mathrm{Cl})\end{array}$} \\
\hline & Baseline & End of treatment & Baseline & End of treatment & \\
\hline $\mathrm{FEV}_{1}$ & $n=19^{*}$ & & $n=20$ & & \\
\hline Measured (L) & $2.10(0.53)$ & $2.05(0.63)$ & $1.99(0.57)$ & $2.07(0.52)$ & $-0.12(-0.25$ to -0.002$) \dagger$ \\
\hline$\%$ predicted & $78.6(15.7)$ & $75.8(18.0)$ & $77.9(19.2)$ & $82.6(19.4)$ & $-7.4(-14.6$ to -0.2$) \dagger$ \\
\hline FVC & $n=19^{*}$ & & $n=20$ & & \\
\hline Measured (L) & $2.57(0.71)$ & $2.48(0.77)$ & $2.50(0.65)$ & $2.53(0.66)$ & $-0.11(-0.21$ to -0.02$)$ \\
\hline$\%$ predicted & $75.0(17.9)$ & $71.1(18.7)$ & $77.9(17.6)$ & $78.1(18.3)$ & $-5.1(-9.4$ to -0.8$)$ \\
\hline $\mathrm{FEV}_{1} / \mathrm{FVC}$ & $n=19^{*}$ & & $n=20$ & & \\
\hline$\%$ & $0.82(0.04)$ & $0.83(0.07)$ & $0.80(0.13)$ & $0.83(0.07)$ & $0.00(-0.04$ to 0.04$)$ \\
\hline Kco & $\mathrm{n}=18^{*}, \ddagger$ & & $\mathrm{n}=18 \S$ & & \\
\hline Measured $(\mathrm{mmol} / \mathrm{min} / \mathrm{kPa} / \mathrm{L})$ & $1.18(0.29)$ & $1.21(0.31)$ & $1.11(0.27)$ & $1.04(0.28)$ & $0.11(0.002$ to 0.22$)$ \\
\hline$\%$ predicted & $90.7(21.7)$ & $94.3(23.1)$ & $85.3(22.6)$ & $80.3(22.7)$ & $9.3(0.9$ to 17.6$)$ \\
\hline TLCo & $\mathrm{n}=18^{*}, \neq$ & & $n=18 \S$ & & \\
\hline Measured $(\mathrm{mmol} / \mathrm{min} / \mathrm{kPa})$ & $4.42(1.60)$ & $4.39(1.53)$ & $3.98(1.28)$ & $3.80(1.43)$ & $0.19(-0.30$ to 0.68$)$ \\
\hline$\%$ predicted & $52.4(15.8)$ & $52.1(14.4)$ & $50.1(15.8)$ & $47.7(16.2)$ & $2.5(-3.1$ to 8.0$)$ \\
\hline 6MWD & $n=19^{*}$ & & $n=199$ & & \\
\hline Measured (metres) & $408.5(102.6)$ & $386.3(130.6)$ & $372.4(112.7)$ & $381.1(109.9)$ & $-30.4(-68.9 \text { to } 8.1)^{* *}$ \\
\hline
\end{tabular}

Data are presented as mean (SD).

${ }^{*}$ Assessments not performed as participant in hospital with sepsis.

tModel has poor fit due to outlying values for one participant.

¥Participant unable to breath-hold, hence gas transfer not measurable.

$\S$ One participant unable to perform full PFTs and one participant had poor technique in lung function at baseline. Unable to measure gas transfer factors.

ๆWalk test not performed due to above knee amputation.

${ }^{* *}$ Model has poor fit due to an outlying value for one participant.

6MWD, 6 min walk distance; TLco, transfer factor for carbon monoxide. 
Table 5 Adverse events (by preferred term) reported in more than one omeprazole participant

\begin{tabular}{|c|c|c|c|c|}
\hline & \multicolumn{2}{|c|}{ Omeprazole $(n=23)$} & \multicolumn{2}{|c|}{ Placebo $(n=22)$} \\
\hline & $\mathrm{n}$ & $\%$ & $\mathrm{n}$ & $\%$ \\
\hline Lower respiratory tract infection & 6 & 26 & 3 & 14 \\
\hline Vomiting & 2 & 9 & 4 & 18 \\
\hline Urinary tract infection & 2 & 9 & 0 & 0 \\
\hline Abdominal pain & 3 & 13 & 3 & 14 \\
\hline Cough & 3 & 13 & 1 & 5 \\
\hline
\end{tabular}

primary clinical outcome data for 40 participants. Future trial design will therefore require to incorporate realistic time scales for recruitment.

Our data suggest that compliance with treatment was good. Non-invasive, portable cough monitoring was readily accepted by participants and there were no technical failures. Patients with IPF find cough intrusive and disabling. ${ }^{2} 35$ Cough would therefore appear to be a worthwhile end point for trials in IPF. However, although average cough frequency was lower in the omeprazole group, no changes were seen in participant-reported cough or reflux questionnaires. The lack of change in reflux symptoms is not especially surprising when those who could not tolerate PPI withdrawal were excluded from further study. While a reduction in objective cough frequency would satisfy the rigour required of an objective outcome measure in RCTs, if patients' experience of cough is apparently unaffected the benefits are harder to envisage. One potential explanation is that the LCQ was never developed for use in patients with IPF and it is likely that it does not capture the true impact of coughing in this patient group, and in particular the interaction between cough and breathlessness. The relationship between objective cough frequency and perceived cough severity is complex. It may be advisable for future, larger trials to incorporate other cough and/or quality of life measures alongside objective cough monitoring, such as cough severity visual analogue scales and a generic quality of life tool.

In contrast to the acceptability of cough monitoring, our data suggest that, in studies primarily focused on cough, invasive oesophageal studies and bronchoscopy were not favoured by participants with IPF. Less than one-third of participants were willing to have these assessments. No reliable trends were observed from the data obtained, although two participants in the omeprazole group had bacterial pathogens cultured from BAL, reinforcing the suggestion that lower respiratory tract infection should be an embedded part of safety assessments in future trials.

Whether we used the most appropriate PPI, in the most appropriate dose, for the most appropriate length of time is open to debate. Twice-daily omeprazole was selected on the basis of good 24 hours acid suppression, ${ }^{36}$ given that omeprazole appears to suppress acid secretion effectively for 12 hours. Omeprazole induces CYP1A2 and could theoretically reduce circulating pirfenidone concentrations (patients in this study were informed of this theoretical risk ahead of randomisation). ${ }^{37}$ Future trials should consider use of alternative PPIs without the potential for drug interaction with pirfenidone, in particular. The 3-month duration of treatment was largely pragmatic.

Whether our participants were representative of 'real-life' IPF is also open to question. Our cohort does seem broadly reflective of patients with IPF recruited to RCTs in terms of mean age and baseline FVC, TLco and 6MWD. We recruited more men than women, in keeping with the sex distribution of IPF, ${ }^{38}$ but our population showed even higher male distribution than in most trials. Nintedanib was only recently available to patients in the UK at the time of the trial, and use in this study was therefore lower than in current practice.

In summary, we believe these data support the pursuit of a larger multicentre trial to determine whether PPIs can reduce cough in IPF and provide a suite of background information to inform rational design of such a trial. Future trials should be large enough to mitigate risks to recruitment and should specifically incorporate assessments of lower respiratory tract infection and lung function as part of safety analysis.

\section{Author affiliations}

${ }^{1}$ Institute of Cellular Medicine, Newcastle University, Newcastle upon Tyne, UK ${ }^{2}$ Institute of Health and Society, Newcastle University, Newcastle upon Tyne, UK ${ }^{3}$ Newcastle Clinical Trials Unit, Newcastle University, Newcastle upon Tyne, UK ${ }^{4}$ Division of Infection, Immunity and Respiratory Medicine, University of Manchester, Manchester, UK

${ }^{5}$ Institute for Cell and Molecular Biosciences, Newcastle University, Newcastle upon Tyne, UK

${ }^{6}$ Northern Oesophago-Gastric Cancer Unit, Newcastle upon Tyne Hospitals NHS Foundation Trust, Newcastle upon Tyne, UK

${ }^{7}$ Manchester University NHS Foundation Trust, Manchester, UK

${ }^{8}$ Department of Respiratory Medicine, Newcastle upon Tyne Hospitals NHS

Foundation Trust, Newcastle upon Tyne, UK

Acknowledgements The authors would like to thank all the trial participants as well as those patients who were assessed for eligibility but not randomised. The authors would also like to thank the members of the study Data Monitoring and Safety Committee and Trial Steering Committee, and the colleagues in the Lung Function Department, Bronchoscopy Unit and Oesophageal Physiology Units, Royal Victoria Infirmary, Newcastle upon Tyne, UK.

Contributors AJS, IAF, CW, VR, JAS, JP and SMG designed the trial; PD and WF recruited patients and coordinated and supervised patient visits; RJ performed oesophageal studies; IAF performed bronchoscopies; SS and JAS performed and analysed cough studies; HM and VR performed statistical analysis; RF provided CTU oversight; CW and JP performed laboratory assays; WF, PD, HM, VR, IAF and AJS wrote the manuscript. All authors approved the manuscript.

Funding The study was funded by a grant from the British Lung Foundation (grant IPFPSG 12-7).

Competing interests JAS is a named inventor on a patent describing methods for detecting cough from sound recordings. The patent is owned by Manchester University Foundation Trust and licensed to Vitalograph, with whom JAS collaborates. She has received no royalties to date. None of the other authors have any interests to declare.

\section{Patient consent Obtained.}

Ethics approval The trial was approved by the Yorkshire and The Humber-Leeds West Research Ethics Committee (13/YH/0284).

Provenance and peer review Not commissioned; externally peer reviewed.

Open access This is an open access article distributed in accordance with the Creative Commons Attribution Non Commercial (CC BY-NC 4.0) license, which permits others to distribute, remix, adapt, build upon this work non-commercially, and license their derivative works on different terms, provided the original work is properly cited, appropriate credit is given, any changes made indicated, and the use is non-commercial. See: http://creativecommons.org/licenses/by-nc/4.0/.

\section{REFERENCES}

1 Horton MR, Santopietro V, Mathew L, et al. Thalidomide for the treatment of cough in idiopathic pulmonary fibrosis: a randomized trial. Ann Intern Med 2012;157:398-406.

2 Key AL, Holt K, Hamilton A, et al. Objective cough frequency in idiopathic pulmonary fibrosis. Cough 2010;6:4.

3 Hope-Gill BD, Hilldrup S, Davies C, et al. A study of the cough reflex in idiopathic pulmonary fibrosis. Am J Respir Crit Care Med 2003;168:995-1002.

4 Ghebre YT, Raghu G. Idiopathic pulmonary fibrosis: novel concepts of proton pump inhibitors as antifibrotic drugs. Am J Respir Crit Care Med 2016;193:1345-52.

5 Savarino E, Carbone R, Marabotto E, et al. Gastro-oesophageal reflux and gastric aspiration in idiopathic pulmonary fibrosis patients. Eur Respir J 2013;42:1322-31.

6 Raghu G, Freudenberger TD, Yang S, et al. High prevalence of abnormal acid gastrooesophageal reflux in idiopathic pulmonary fibrosis. Eur Respir J 2006;27:136-42. 
7 Tobin RW, Pope CE, Pellegrini CA, et al. Increased prevalence of gastroesophageal reflux in patients with idiopathic pulmonary fibrosis. Am J Respir Crit Care Med 1998;158:1804-8.

8 Raghu G, Rochwerg B, Zhang Y, et al. An Official ATS/ERS/JRS/ALAT Clinical Practice Guideline: Treatment of idiopathic pulmonary fibrosis. An Update of the 2011 Clinical Practice Guideline. Am J Respir Crit Care Med 2015;192:e3-e19.

9 Raghu G, Collard HR, Egan JJ, et al. An official ATS/ERS/JRS/ALAT statement: idiopathic pulmonary fibrosis: evidence-based guidelines for diagnosis and management. Am J Respir Crit Care Med 2011;183:788-824.

10 Richeldi L, Collard HR, Jones MG. Idiopathic pulmonary fibrosis. Lancet 2017;389:1941-52.

11 Demeester TR, Johnson LF, Joseph GJ, et al. Patterns of gastroesophageal reflux in health and disease. Ann Surg 1976;184:459-70.

12 Belafsky PC, Postma GN, Koufman JA. Validity and reliability of the reflux symptom index (RSI). J Voice 2002;16:274-7.

13 Eypasch E, Williams JI, Wood-Dauphinee S, et al. Gastrointestinal Quality of Life Index: development, validation and application of a new instrument. Br J Surg $1995 ; 82: 216-22$.

14 Birring SS, Prudon B, Carr AJ, et al. Development of a symptom specific health status measure for patients with chronic cough: Leicester Cough Questionnaire (LCQ). Thorax 2003;58:339-43.

15 McGuinness K, Holt K, Dockry R, et al. Validation of the VitaloJAKTM 24 Hour Ambulatory Cough Monitor. Thorax 2012;67:A131.

16 Barton A, Gaydecki P, Holt K, et al. Data reduction for cough studies using distribution of audio frequency content. Cough 2012;8:12.

17 Hertzog MA. Considerations in determining sample size for pilot studies. Res Nurs Health 2008;31:180-91.

18 Lancaster GA, Dodd S, Williamson PR. Design and analysis of pilot studies: recommendations for good practice. J Eval Clin Pract 2004;10:307-12.

19 King TE, Bradford WZ, Castro-Bernardini S, et al. A phase 3 trial of pirfenidone in patients with idiopathic pulmonary fibrosis. N Eng/ J Med 2014;370:2083-92.

20 Richeldi L, du Bois RM, Raghu G, et al. Efficacy and safety of nintedanib in idiopathic pulmonary fibrosis. N Engl J Med 2014;370:2071-82.

21 Lutherer LO, Nugent KM, Schoettle BW, et al. Low-dose oral interferon $\alpha$ possibly retards the progression of idiopathic pulmonary fibrosis and alleviates associated cough in some patients. Thorax 2011;66:446-7.

22 van Manen MJ, Birring SS, Vancheri C, et al. Cough in idiopathic pulmonary fibrosis. Eur Respir Rev 2016;25:278-86.

23 Vigeland $\mathrm{CL}$, Hughes $\mathrm{AH}$, Horton MR. Etiology and treatment of cough in idiopathic pulmonary fibrosis. Respir Med 2017;123:98-104.
24 Lee JS, Ryu JH, Elicker BM, et al. Gastroesophageal reflux therapy is associated with longer survival in patients with idiopathic pulmonary fibrosis. Am J Respir Crit Care Med 2011;184:1390-4.

25 Lee JS, Collard HR, Anstrom KJ, et al. Anti-acid treatment and disease progression in idiopathic pulmonary fibrosis: an analysis of data from three randomised controlled trials. Lancet Respir Med 2013;1:369-76.

26 Kreuter M, Wuyts W, Renzoni E, et al. Antacid therapy and disease outcomes in idiopathic pulmonary fibrosis: a pooled analysis. Lancet Respir Med 2016;4:381-9.

27 Kreuter M, Spagnolo P, Wuyts W, et al. Antacid therapy and disease progression in patients with idiopathic pulmonary fibrosis who received pirfenidone. Respiration 2017:93:415-23.

28 Raghu G, Pellegrini CA, Yow E, et al. Laparoscopic anti-reflux surgery for the treatment of idiopathic pulmonary fibrosis (WRAP-IPF): a multicentre, randomised, controlled phase 2 trial. Lancet Respir Med 2018;6:707-14.

29 Kelsall A, Houghton LA, Jones $H$, et al. A novel approach to studying the relationship between subjective and objective measures of cough. Chest 2011;139:569-75

30 Birring SS, Wijsenbeek MS, Agrawal S, et al. A novel formulation of inhaled sodium cromoglicate (PA101) in idiopathic pulmonary fibrosis and chronic cough: a randomised, double-blind, proof-of-concept, phase 2 trial. Lancet Respir Med 2017:5:806-15.

31 van Manen MJG, Birring SS, Vancheri C, et al. Effect of pirfenidone on cough in patients with idiopathic pulmonary fibrosis. Eur Respir J 2017;50:1701157.

32 Laheij RJ, Sturkenboom MC, Hassing RJ, et al. Risk of community-acquired pneumonia and use of gastric acid-suppressive drugs. JAMA 2004;292:1955-60.

33 Eom CS, Jeon CY, Lim JW, et al. Use of acid-suppressive drugs and risk of pneumonia: a systematic review and meta-analysis. CMAJ 2011;183:310-9.

34 Lambert AA, Lam JO, Paik JJ, et al. Risk of community-acquired pneumonia with outpatient proton-pump inhibitor therapy: a systematic review and meta-analysis. PLoS One 2015;10:e0128004.

35 Swigris JJ, Stewart AL, Gould MK, et al. Patients' perspectives on how idiopathic pulmonary fibrosis affects the quality of their lives. Health Qual Life Outcomes 2005:3:61.

36 Miner P, Katz PO, Chen Y, et al. Gastric acid control with esomeprazole, lansoprazole, omeprazole, pantoprazole, and rabeprazole: a five-way crossover study. Am J Gastroenterol 2003;98:2616-20

37 Rost KL, Fuhr U, Thomsen T, et al. Omeprazole weakly inhibits CYP1A2 activity in man Int J Clin Pharmacol Ther 1999;37:567-74.

38 Navaratnam V, Fleming KM, West J, et al. The rising incidence of idiopathic pulmonary fibrosis in the U.K. Thorax 2011;66:462-7. 